\title{
The Displacement and Strain Field of Three-dimensional Rheologic Model of Earthquake Preparation
}

\author{
Zhiping Song, ${ }^{1}$ Xiangchu Yin, ${ }^{2}$ Shirong Mei, ${ }^{2}$ Yucang Wang, ${ }^{3}$ Can Yin, ${ }^{3}$ \\ Huinui Zhang, ${ }^{4}$ and LANGPING $\mathrm{ZHANG}^{4}$
}

\begin{abstract}
Based on the three-dimensional elastic inclusion model proposed by Dobrovolskii, we developed a rheological inclusion model to study earthquake preparation processes. By using the Corresponding Principle in the theory of rheologic mechanics, we derived the analytic expressions of viscoelastic displacement $U(r, t), V(r, t)$ and $W(r, t)$, normal strains $\varepsilon_{x x}(r, t), \varepsilon_{y y}(r, t)$ and $\varepsilon_{z z}(r, t)$ and the bulk strain $\theta(r, t)$ at an arbitrary point $(x, y, z)$ in three directions of $X$ axis, $Y$ axis and $Z$ axis produced by a three-dimensional inclusion in the semi-infinite rheologic medium defined by the standard linear rheologic model. Subsequent to the spatial-temporal variation of bulk strain being computed on the ground produced by such a spherical rheologic inclusion, interesting results are obtained, suggesting that the bulk strain produced by a hard inclusion change with time according to three stages $(\alpha, \beta, \gamma)$ with different characteristics, similar to that of geodetic deformation observations, but different with the results of a soft inclusion. These theoretical results can be used to explain the characteristics of spatial-temporal evolution, patterns, quadrant-distribution of earthquake precursors, the changeability, spontaneity and complexity of short-term and imminent-term precursors. It offers a theoretical base to build physical models for earthquake precursors and to predict the earthquakes.
\end{abstract} sions.

Key words: Three-dimensional elastic inclusion mode, rheological inclusion model, analytic expres-

\section{Introduction}

The studies of the inclusion model started in the 1950s. EsHeLBy (1957) first studied an inhomogeneous problem in which a region (or 'inclusion') with homogeneous elastic constants was embedded within a matrix with different

\footnotetext{
${ }^{1}$ Earthquake Administration of Shanghai Municipality, Shanghai 200062, China. E-mail: zpsong@sohu.com

${ }^{2}$ Institue of Earthquake Prediction, China Earthquake Administration, Beijing 100036, China.

${ }^{3}$ Quakes, Earth Systems Science Computational Centre, The University of Queensland, St. Lucia, Brisbane, QLD 4072, Australia.

${ }^{4}$ State Key Laboratory of Nonlinear Mechanics, Institute of Mechanics, Chinese Academy of Sciences, Beijing 100080, China.
} 
elastic constants and derived the elastic stresses in matrix and inclusion. Eshelby studied two kinds of inclusions, which were the hard inclusion in which the elastic modulus of inclusion is higher that of the matrix, and the soft inclusion in which the elastic modulus of inclusion is lower. Eshelby also studied the elliptic sphere inclusion model in the elastic medium and gave analytical solutions of the stress field of inclusion and matrix and applied to some problems of physics and mechanics.

BRADY (1974) proposed the soft inclusion model. The main hypothesis is that the intense crack concentrations developed within localized regions when a rock specimen is close to ultimate failure can be represented by inclusions of lower modulus. He proposed a scale-independent theory of rock failure, and studied the inclusion of crustal and deep earthquakes, and gave meticulous explanations for short-term premonitory phenomena of earthquakes. He pointed out that for the process of earthquake failures, it is appropriate to use the soft inclusion rather than the hard one.

DobrovolskiI (1991) further studied the hard inclusion model on the basis of elastic mechanics. He gave the elastic analytical solutions of the displace, the strain, the geo-electricity and the geomagnetism of random point $(x, y, z)$ produced by an earthquake generating body of a three-dimension inclusion (spherical inclusion or rotating spheroid). He discussed the precursors' mechanism of seismicity, deformation, water-radon, geomagnetism, geo-electricity and so on and the relation between the distributive range of earthquake precursors and the magnitude of the earthquake. In fact, this is the first excellent model that remarks on features of long-term precursor fields. However it is still difficult to explain the mechanism of short-term and immediate precursors by using this model.

MEI (1994) set up the strong-body earthquake-generating model based on geophysics. By studying the tomographic image, it has been affirmed that the existence of a hard inclusion is an important condition for the high concentration of large amounts of strain energy. Numerous theoretical and experimental studies have consistently indicated that rock instability, sudden fracture and stress drop would be possible only if the stiffness of the source body is greater than the environmental stiffness.

They viewed the generating body as a three-dimension inclusion. Eshelby gave analytical solutions of the stress field of different inclusions. Brady proposed the soft inclusion model to explain short-term premonitory phenomena of earthquakes. Dobrovolskii studied the hard inclusion model to study long-term precursors. All of these models are elastic inclusion models. Rocks exhibit mainly elastic behaviors under low temperature, low pressure and short-time external forces, but they exhibit rheologic behavor under high temperature, high pressure and long-time external forces. Therefore it is appropriate that earthquake rupture process could be analyzed using the elastic theory, considering the fact that it occurs within seconds to hundreds of seconds. However, for the earthquake 
generating process lasting over hundreds or thousand of years, the rheologic property of rock must be taken into account (ZHANG et al., 1987; YIN et al., 1982; MeI et al., 1993).

Our previous work extended Dobrovolskii's elastic theory of the hard inclusion model into the rheologic inclusion model (SonG, 1996). We analyzed preliminarily the spatial and temporal evolution process of bulk strain field for this model (SoNG et al., 2000). In this paper we give the displacement field and the strain field of the rheologic inclusion model, and endeavor to discuss the characteristics of spatialtemporal evolution, patterns and quadrant-distribution of earthquake precursors. Additional and detailed derivations can be found in two papers (Song et al., 2003, 2004).

\section{Basic Theory}

Assume there is an inclusion in the semi-infinite medium (Fig. 1a). $S_{0}$ is the surface of the Earth where the effects of the atmosphere pressure ignored. $S_{1}$ is a hypothetical surface of the Earth and its boundary condition may be given in terms of displacement, stress or other physical parameters. With the first-order approximation, the external forces for a shallow earthquake may include body forces, whereas the gravity and the tidal force may be neglected. Although the tidal disturbing effect is small, it may be significant due to its long and periodical nature, therefore in detailed analysis this effect should be considered.

In a rectangular coordinate system, the plane $X O Y$ is in the surface of the Earth. The $Z$-axis points to the center of the Earth. The center of the inclusion is at the point $(0,0, \mathrm{H})$. The boundary condition is that except for $\sigma_{x y}^{\infty}=\sigma_{y x}^{\infty}=-\tau$ (Fig. 1), all other
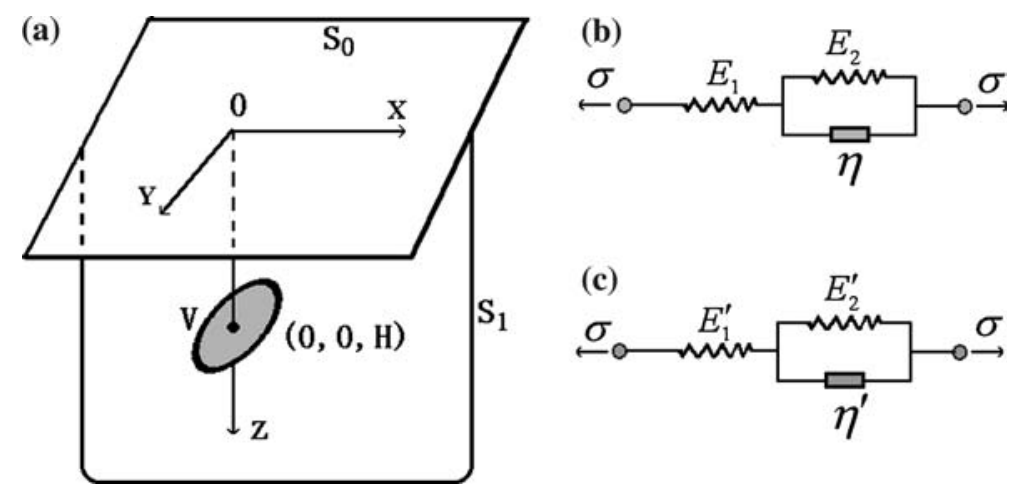

Figure 1

Three-dimensional inclusion and its rheologic medium model. (a) Three-dimensional inclusion model. (b) Rheologic medium model outside the inclusion. (c) Rheologic medium model inside the inclusion. 
components are zero. That is, uniform shear stresses are applied at the infinitude and the bottom is a free surface. If the boundary condition is a function of time, it is expressed as $\tau H(t)$, where $H(t)$ is a unit jump function. Therefore, we choose the standard linear solid model (series connection between a spring and a Kelvin body or parallel connection between a spring and a Maxwell body) (YIN et al., 1982) as the medium model inside and outside the three-dimensional inclusion in Figures.1b and 1c (Song et al., 2003).

On the basis of Dobrovolskii's hard inclusion elastic theory and the Corresponding Principle of rheologic mechanics (YIN, 1985), we derived the viscoelastic expression of displacement produced by the inclusion in the rheologic medium.

From the analytic expression $U_{i}^{e}(r)$ of displacement of an elastic inclusion, we can derive the solution $\bar{U}_{i}^{e}(r, s)$ of elastic displacement in the image-plane by using Laplace Transform. Then by substituting the elastic parameters $\mu$ and $K$ with $\bar{\mu}(s)$ and $\bar{K}(s)$ of the image-plane, we can derive the solution $\bar{U}_{i}^{v}(r, s)$ of visco-elastic displacement in the image-plane. At last, using the Inverse Laplace Transform, the viscoelastic expression $U_{i}^{v}(r, t)$ can be obtained.

\section{Displacement Field}

The analytic solution of visco-elastic displacement $U(r, t)$ in the $X$ axis, $V(r, t)$ in the $Y$ axis and $W(r, t)$ in the $Z$-axis has been obtained in SoNG et al. (2003).

For example, the analytic solution of visco-elastic displacement $U(r, t)$ in the $X$ axis of a point $(x, y, z)$ is as follows:

$$
\begin{aligned}
U(r, t)= & \frac{V y \tau}{8 \pi}\left\{G_{u 0} H(t)+\left(G_{u 1}+G_{u 1}^{\prime \prime}+t G_{u 1}^{\prime}\right) \exp \left(-\frac{q_{0}}{q_{1}} t\right)+G_{u 2} \exp \left(-\frac{1}{p_{1}^{\prime}} t\right)\right. \\
& \left.+G_{u 3} \exp \left(-\frac{3 K+2 q_{0}}{3 K p_{1}+2 q_{1}} t\right)+G_{u 4} \exp \left(-\frac{6 K+q_{0}}{6 K p_{1}+q_{1}} t\right)\right\}
\end{aligned}
$$

Defining the time parameters $T_{1}, T_{2}, T_{3}, T_{4}$ as

$$
T_{1}=\frac{q_{0}}{q_{1}}, \quad T_{2}=\frac{1}{p_{1}^{\prime}}, \quad T_{3}=\frac{3 K+2 q_{0}}{3 K p_{1}+2 q_{1}}, \quad T_{4}=\frac{6 K+q_{0}}{6 K p_{1}+q_{1}} .
$$

The Parameters $G_{u i}$ in Equation (1) are 


$$
\begin{aligned}
& \left(G_{u 0}=2\left(\frac{q_{0}^{\prime}}{q_{0}}-1\right) \cdot\left[\frac{S_{u}(0)}{q_{0}\left(3 K+q_{0}\right)}+P_{u 2} \cdot \frac{3}{3 K+2 q_{0}}+P_{u 4} \cdot \frac{3}{6 K+q_{0}}\right]\right. \\
& G_{u 1}=\frac{2\left(1-p_{1} T_{1}\right)}{q_{0}}\left\{\begin{array}{c}
\frac{S_{u}\left(T_{1}\right)}{\left(3 K+2 q_{0}\right)-\left(3 K p_{1}+2 q_{1}\right) T_{1}}-\frac{3\left(1-p_{1} T_{1}\right)\left(q_{0}^{\prime}-q_{1}^{\prime} T_{1}\right)}{1-p_{1}^{\prime} T_{1}} \bullet \\
{\left[\frac{P_{u 2}}{\left(3 K+2 q_{0}\right)-\left(3 K p_{1}+2 q_{1}\right) T_{1}}+\frac{P_{u 4}}{\left(6 K+q_{0}\right)-\left(6 K p_{1}+q_{1}\right) T_{1}}\right]}
\end{array}\right\} \\
& G_{u 2}=-2\left(1-p_{1} T_{2}\right)^{2} \\
& \left\{\times \frac{q_{0}^{\prime}-q_{1}^{\prime} T_{2}}{q_{0}-q_{1} T_{2}}\left\{\begin{array}{c}
\frac{S_{u}\left(T_{2}\right)}{\left(q_{0}-q_{1} T_{2}\right)\left[\left(3 K+2 q_{0}\right)-\left(3 K p_{1}+2 q_{1}\right) T_{2}\right]} \\
+\frac{3 P_{u 2}}{\left(3 K+2 q_{0}\right)-\left(3 K p_{1}+2 q_{1}\right) T_{1}}+\frac{3 P_{u 4}}{\left(6 K+q_{0}\right)-\left(6 K p_{1}+q_{1}\right) T_{1}}
\end{array}\right\}\right. \\
& G_{u 3}=-\frac{2\left(1-p_{1} T_{3}\right)}{3 K+2 q_{0}}\left[\frac{1-p_{1} T_{3}}{1-p_{1}^{\prime} T_{3}} \cdot \frac{q_{0}^{\prime}-q_{1}^{\prime} T_{3}}{q_{0}-q_{1} T_{3}}-1\right]\left[\frac{S_{u}\left(T_{3}\right)}{q_{0}-q_{1} T_{3}}+3 P_{u 2}\right] \\
& G_{u 4}=-\frac{6\left(1-p_{1} T_{4}\right)}{6 K+q_{0}}\left[\frac{1-p_{1} T_{4}}{1-p_{1}^{\prime} T_{4}} \cdot \frac{q_{0}^{\prime}-q_{1}^{\prime} T_{4}}{q_{0}-q_{1} T_{4}}-1\right] \cdot P_{u 4} \\
& G_{u 1}^{\prime}=\frac{-2\left(q_{0}^{\prime}-q_{1}^{\prime} T_{1}\right)\left(1-p_{1} T_{1}\right)^{2}}{q_{0} q_{1}\left(1-p_{1}^{\prime} T_{1}\right)\left[\left(3 K+2 q_{0}\right)-\left(3 K p_{1}+2 q_{1}\right) T_{1}\right]} \cdot S_{u}\left(T_{1}\right) \\
& G_{u 1}^{\prime}=2\left\{P_{u 1} \cdot S\left(6 K+q_{0}, 6 K p_{1}+q_{1}\right)+P_{u 3} \cdot S\left(6 K+7 q_{0}, 6 K p_{1}+7 q_{1}\right)\right\}
\end{aligned}
$$

where

$$
S_{u}(T)=P_{u 1} \cdot\left[6 K+q_{0}-\left(6 K p_{1}+q_{1}\right) T\right]+P_{u 3} \cdot\left[6 K+7 q_{0}-\left(6 K p_{1}+7 q_{1}\right) T\right] .
$$

The universal function $S(m, n)$ is

$$
\begin{aligned}
S(m, n)= & {\left[A A\left(3 K+2 q_{0}, 3 K p_{1}+2 q_{1}\right)+B B\left(3 K+2 q_{0}, 3 K p_{1}+2 q_{1}\right)\right] \cdot\left(m-n T_{1}\right) } \\
& +C C\left(3 K+2 q_{0}, 3 K p_{1}+2 q_{1}\right) \cdot n .
\end{aligned}
$$

In Equation (4), $A A(m, n), B B(m, n), C C(m, n)$ and $D D(m, n)$ are

$$
\left\{\begin{array}{l}
A A(m, n)=-\frac{\left(1-p_{1} T_{1}\right)\left[q_{1}^{\prime}\left(1-p_{1} T_{1}\right)-2 p_{1}\left(q_{0}^{\prime}-q_{1}^{\prime} T_{1}\right)\right]}{q_{0} q_{1}\left(1-p_{1}^{\prime} T_{1}\right)} \cdot \frac{1}{m-n T_{1}} \\
B B(m, n)=-\frac{\left(1-p_{1} T_{1}\right)^{2}\left(q_{0}^{\prime}-q_{1}^{\prime} T_{1}\right)}{q_{0}\left(1-p_{1}^{\prime} T_{1}\right)} \cdot \frac{D D(m, n)}{m-n T_{1}} \\
C C(m, n)=-\frac{\left(1-p_{1} T_{1}\right)^{2}\left(q_{0}^{\prime}-q_{1}^{\prime} T_{1}\right)}{q_{0} q_{1}\left(1-p_{1}^{\prime} T_{1}\right)} \cdot \frac{1}{m-n T_{1}} \\
D D(m, n)=\frac{1}{q_{0}}-\frac{p_{1}^{\prime}}{q_{1}\left(1-p_{1}^{\prime} T_{1}\right)}-\frac{n}{q_{1}\left(m-n T_{1}\right)}
\end{array}\right.
$$


The parameters related to the coordinate system are

$$
\left\{\begin{array}{l}
P_{u 1}=-\frac{6 H z}{r_{2}^{5}}+x^{2}\left(-\frac{3}{r_{1}^{5}}+\frac{30 H z}{r_{2}^{7}}\right) \\
P_{u 2}=\frac{1}{r_{2}^{3}}-\frac{1}{r_{1}^{3}} \\
P_{u 3}=-\frac{3 x^{2}}{r_{2}^{5}} \\
P_{u 4}=-\frac{4}{r_{2}\left(r_{2}+H+z\right)^{2}}+\frac{4\left(3 r_{2}+H+z\right)}{r_{2}^{3}\left(r_{2}+H+z\right)^{3}} \cdot x^{2}
\end{array}\right.
$$

When $t=0$, the displacement $U(r, 0)$ in the $X$ axis is

$$
U(r, 0)=\frac{V y \tau}{8 \pi}\left\{G_{u 0}+G_{u 1}+G_{u 1}^{\prime \prime}+G_{u 2}+G_{u 3}+G_{u 4}\right\}
$$

The analytic solution of visco-elactic displacement $V(r, t)$ in the $Y$ axis of the point $(x, y, z)$ is as follows:

$$
\begin{aligned}
V(r, t)= & \frac{V x \tau}{8 \pi}\left\{G_{v 0} H(t)+\left(G_{v 1}+G_{v 1}^{\prime \prime}+t G_{v 1}^{\prime}\right) \exp \left(-\frac{q_{0}}{q_{1}} t\right)+G_{v 2} \exp \left(-\frac{1}{p_{1}^{\prime}} t\right)\right. \\
& \left.+G_{v 3} \exp \left(-\frac{3 K+2 q_{0}}{3 K p_{1}+2 q_{1}} t\right)+G_{v 4} \exp \left(-\frac{6 K+q_{0}}{6 K p_{1}+q_{1}} t\right)\right\}
\end{aligned}
$$

where the parameters $G_{v i}(i=0,1,2,3,4)$ are $G_{u i}$ in the Equations (2) (6) by substituting the parameters $P_{u i}$ with $P_{v i}$.

When $t=0$, the displacement $V(r, 0)$ in the $Y$ axis is

$$
V(r, 0)=\frac{V x \tau}{8 \pi}\left\{G_{v 0}+G_{v 1}+G_{v 1}^{\prime \prime}+G_{v 2}+G_{v 3}+G_{v 4}\right\}
$$

The analytic solution of viscoelastic displacement $W(r, t)$ in the $Z$ direction of the points $(x, y, z)$ was obtained.

$$
\begin{aligned}
W(r, t)= & \frac{V \tau x y}{8 \pi}\left\{G_{w 0} H(t)+\left(G_{w 1}+G_{w 1}^{\prime \prime}+t G_{w 1}^{\prime}\right) \exp \left(-\frac{q_{0}}{q_{1}} t\right)+G_{w 2} \exp \left(-\frac{1}{p_{1}^{\prime}} t\right)\right. \\
& \left.+G_{w 3} \exp \left(-\frac{3 K+2 q_{0}}{3 K p_{1}+2 q_{1}} t\right)+G_{w 4} \exp \left(\frac{6 K+q_{0}}{6 K p_{1}+q_{1}} t\right)\right\}
\end{aligned}
$$


where parameters $G_{w i}(i=0,1,2,3,4)$ are as follows

$$
\left\{\begin{aligned}
G_{w 0}= & 2\left(\frac{q_{0}^{\prime}}{q_{0}}-1\right) \cdot\left[\frac{S_{u}(0)}{q_{0}\left(3 K+q_{0}\right)}+\frac{3 P_{w 3}}{6 K+q_{0}}\right] \\
G_{w 1}= & \frac{2\left(1-p_{1} T_{1}\right)}{q_{0}}\left\{\frac{S_{w}\left(T_{1}\right)}{\left(3 K+2 q_{0}\right)-\left(3 K p_{1}+2 q_{1}\right) T_{1}}\right. \\
G_{w 2}= & -2\left(1-p_{1} T_{2}\right)^{2} \frac{q_{0}^{\prime}-q_{1}^{\prime} T_{2}}{q_{0}-q_{1} T_{2}} \\
& \times\left\{\frac{\left(1-p_{1} T_{1}\right)}{1-p_{1}^{\prime} T_{1}} \cdot \frac{3\left(q_{0}^{\prime}-q_{1}^{\prime} T_{1}\right) P_{w 3}}{\left(6 K+q_{0}\right)-\left(6 K p_{1}+q_{1}\right) T_{1}}\right\} \\
G_{w 3}= & -\frac{S_{w}\left(T_{2}\right)}{\left(3 K+2 q_{1} T_{2}\right)\left[\left(3 K+2 q_{0}\right)-\left(3 K p_{1}+2 q_{1}\right) T_{2}\right]}+\frac{\left(6 K+q_{0}\right)-\left(6 K p_{1}+q_{1}\right) T_{2}}{\left(q_{0}-q_{1} T_{3}\right)}\left[\frac{1-p_{1} T_{3}}{1-p_{1}^{\prime} T_{3}} \cdot \frac{q_{0}^{\prime}-q_{1}^{\prime} T_{3}}{q_{0}-q_{1} T_{3}}-1\right] \cdot S_{w}\left(T_{3}\right) \\
G_{w 4}= & -\frac{6\left(1-p_{1} T_{4}\right)}{6 K+q_{0}}\left[\frac{1-p_{1} T_{4}}{1-p_{1}^{\prime} T_{4}} \cdot \frac{q_{0}^{\prime}-q_{1}^{\prime} T_{4}}{q_{0}-q_{1} T_{4}}-1\right] \cdot P_{w 3} \\
G_{w 1}^{\prime}= & -\frac{2\left(q_{0}^{\prime}-q_{1}^{\prime} T_{1}\right)\left(1-p_{1} T_{1}\right)^{2}}{q_{0} q_{1}\left(1-p_{1}^{\prime} T_{1}\right)\left[\left(3 K+2 q_{0}\right)-\left(3 K p_{1}+2 q_{1}\right) T_{1}\right]} \cdot S_{w}\left(T_{1}\right) \\
G_{w 1}^{\prime}= & 2\left\{P_{w 1} \cdot S\left(6 K+q_{0}, 6 K p_{1}+q_{1}\right)+P_{w 2} \cdot S\left(6 K+7 q_{0}, 6 K p_{1}+7 q_{1}\right)\right\} \\
& -11)
\end{aligned}\right.
$$

where

$$
\left\{\begin{array}{l}
P_{w 1}=-\frac{3(H-z)}{r_{1}^{5}}+\frac{30 H z(H+z)}{r_{2}^{7}} \\
P_{w 2}=\frac{3(H-z)}{r_{2}^{5}} \\
P_{w 3}=-\frac{4\left(2 r_{2}+H+z\right)}{r_{2}^{3}\left(r_{2}+H+z\right)^{2}}
\end{array}\right\}
$$

For the universal function $S(n, m)$ see Equation (4). When $t=0$, the displacement $W(r, 0)$ in the $Z$ axis is given by

$$
W(r, 0)=\frac{V \tau x y}{8 \pi}\left\{G_{w 0}+G_{w 1}+G_{w 1}^{\prime \prime}+G_{w 2}+G_{w 3}+G_{w 4}\right\} .
$$




\section{Strain Field}

The normal strain $\varepsilon_{x x}(r, t)$ in $X$ axis, $\varepsilon_{y y}(r, t)$ in $Y$ axis, $\varepsilon_{z z}(r, t)$ in $Z$ axis and then the bulk strain $\theta(r, t)$ has been obtained by SoNG (1996).

The normal strain variation with time for the rheologic incluson model in $X$ axis is (SoNG et al., 2004)

$$
\begin{aligned}
\varepsilon_{x x}(r, t)= & \frac{3 V \tau x y}{8 \pi}\left\{M_{x 0} H(t)+\left(M_{x 1}+M_{x 1}^{\prime \prime}+t M_{x 1}^{\prime}\right) \exp \left(-\frac{q_{0}}{q_{1}} t\right)+M_{x 2} \exp \left(-\frac{1}{p_{1}^{\prime}} t\right)\right. \\
& \left.+M_{x 3} \exp \left(-\frac{3 K+2 q_{0}}{3 K p_{1}+2 q_{1}} t\right)+M_{x 4} \exp \left(-\frac{6 K+q_{0}}{6 K p_{1}+q_{1}} t\right)\right\}
\end{aligned}
$$

The parameters $M_{x i}(i=1,4)$ are as follows:

$$
\left\{\begin{aligned}
& M_{x 0}= 2\left(\frac{q_{0}^{\prime}}{q_{0}}-1\right) \cdot\left[\frac{S_{\varepsilon x x}(0)}{q_{0}\left(3 K+q_{0}\right)}+\cdot \frac{3 P_{x 5}}{6 K+q_{0}}\right] \\
& M_{x 1}= \frac{2\left(1-p_{1} T_{1}\right)}{q_{0}} \\
& \times\left\{\frac{S_{\varepsilon x x}\left(T_{1}\right)}{\left(3 K+2 q_{0}\right)-\left(3 K p_{1}+2 q_{1}\right) T_{1}}-\frac{\left(1-p_{1} T_{1}\right)}{1-p_{1}^{\prime} T_{1}} \cdot \frac{3\left(q_{0}^{\prime}-q_{1}^{\prime} T_{1}\right) P_{x 5}}{\left(6 K+q_{0}\right)-\left(6 K p_{1}+q_{1}\right) T_{1}}\right\} \\
& M_{x 2}=-2\left(1-p_{1} T_{2}\right)^{2} \frac{q_{0}^{\prime}-q_{1}^{\prime} T_{2}}{q_{0}-q_{1} T_{2}} \\
& \times\left\{\frac{S_{\varepsilon x x}\left(T_{2}\right)}{\left(q_{0}-q_{1} T_{2}\right)\left[\left(3 K+2 q_{0}\right)-\left(3 K p_{1}+2 q_{1}\right) T_{2}\right]}+\frac{\left.2 K+q_{0}\right)-\left(6 K p_{1}+q_{1}\right) T_{2}}{(6 K}\right\} \\
& M_{x 3}=-\frac{2\left(1-p_{1} T_{3}\right)}{\left(3 K+2 q_{0}\right)\left(q_{0}-q_{1} T_{3}\right)}\left[\frac{1-p_{1} T_{3}}{1-p_{1}^{\prime} T_{3}} \cdot \frac{q_{0}^{\prime}-q_{1}^{\prime} T_{3}}{q_{0}-q_{1} T_{3}}-1\right] \cdot S_{\varepsilon x x}\left(T_{3}\right) \\
& M_{x 4}=-\frac{6\left(1-p_{1} T_{4}\right)}{6 K+q_{0}}\left[\frac{1-p_{1} T_{4}}{1-p_{1}^{\prime} T_{4}} \cdot \frac{q_{0}^{\prime}-q_{1}^{\prime} T_{4}}{q_{0}-q_{1} T_{4}}-1\right] \cdot P_{x 5} \\
& 2\left(q_{0}^{\prime}-q_{1}^{\prime} T_{1}\right)\left(1-p_{1} T_{1}\right)^{2} \\
& M_{x 1}^{\prime}=-\frac{3 P_{x 5}}{q_{0} q_{1}\left(1-p_{1}^{\prime} T_{1}\right)\left[\left(3 K+2 q_{0}\right)-\left(3 K p_{1}+2 q_{1}\right) T_{1}\right]} \cdot S_{\varepsilon x x}\left(T_{1}\right) \\
& M_{x 1}^{\prime \prime}= 2 \cdot\left\{\begin{array}{c}
P_{x 1} \cdot S\left(6 K+q_{0}, 6 K p_{1}+q_{1}\right)+P_{x 2} \cdot S\left(6 K+7 q_{0}, 6 K p_{1}+7 q_{1}\right) \\
+P_{x 3} \cdot S\left(12 K-q_{0}, 12 K p_{1}-q_{1}\right)+P_{x 4} \cdot S\left(12 K+17 q_{0}, 12 K p_{1}+17 q_{1}\right)
\end{array}\right\} .
\end{aligned}\right.
$$

In the Equation (16), $S_{\varepsilon x x}(T)$ is given by

$$
\begin{aligned}
S_{\varepsilon x x}(T)= & P_{x 1} \cdot\left[6 K+q_{0}-\left(6 K p_{1}+q_{1}\right) T\right]+P_{x 2} \cdot\left[6 K+7 q_{0}-\left(6 K p_{1}+7 q_{1}\right) T\right] \\
& +P_{x 3} \cdot\left[12 K-q_{0}-\left(12 K p_{1}-q_{1}\right) T\right]+P_{x 4} \cdot\left[12 K+17 q_{0}-\left(12 K p_{1}+17 q_{1}\right) T\right] .
\end{aligned}
$$


For the universal function $S(n, m)$ in Equation (16), see Equations (4) and (5). The parameters $P_{x i}(\mathrm{i}=1,4)$ in Equations $(16)$ and $(17)$ are given by

$$
\left\{\begin{array}{l}
P_{x 1}=\frac{30 H z}{r_{2}^{7}}+x^{2}\left(\frac{5}{r_{1}^{7}}-\frac{70 H z}{r_{2}^{9}}\right) \\
P_{x 2}=\frac{5}{r_{2}^{7}} \cdot x^{2} \\
P_{x 3}=-\frac{1}{r_{1}^{5}} \\
P_{x 4}=-\frac{1}{r_{2}^{5}} \\
P_{x 5}=\frac{4\left(3 r_{2}+H+z\right)}{r_{2}^{3}\left(r_{2}+H+z\right)^{3}}-\frac{4 x^{2}}{r_{2}^{5}\left(r_{2}+H+z\right)^{2}}-\frac{8\left(2 r_{2}+H+z\right)}{r_{2}^{4}\left(r_{2}+H+z\right)^{4}} \cdot x^{2} .
\end{array}\right.
$$

In Equation (15), when $t=0$, the normal strain $\varepsilon \hat{x x}(r, 0)$ in $X$ axis is

$$
\varepsilon_{x x}(r, 0)=\frac{3 V \tau x y}{8 \pi}\left\{M_{x 0}+M_{x 1}+M_{x 1}^{\prime \prime}+M_{x 2}+M_{x 3}+M_{x 4}\right\} .
$$

The analytic expression of the viscoelastic normal strain $\varepsilon_{y y}(r, t)$ at a point $(x, y, z)$ in $Y$ axis is

$$
\begin{aligned}
\varepsilon_{y y}(r, t)= & \frac{3 V \tau x y}{8 \pi}\left\{M_{y 0} H(t)+\left(M_{y 1}+M_{y 1}^{\prime \prime}+t \cdot M_{y 1}^{\prime}\right) \exp \left(-\frac{q_{0}}{q_{1}} t\right)+M_{y 2} \exp \left(-\frac{1}{p_{1}^{\prime}} t\right)\right. \\
& \left.+M_{y 3} \exp \left(-\frac{3 K+2 q_{0}}{3 K p_{1}+2 q_{1}} t\right)+M_{y 4} \exp \left(-\frac{6 K+q_{0}}{6 K p_{1}+q_{1}} t\right)\right\}
\end{aligned}
$$

where the parameters $M_{y i}$ are the parameters $M_{x i}$ in Equations (16) and (17) by substituting $P_{x i}$ with $P_{y i}$. Of these, the parameters $P_{y i}$ which related to the coordinate system are given by

$$
\left\{\begin{array}{l}
P_{y 1}=-\frac{30 H z}{r_{2}^{7}}+y^{2}\left(\frac{5}{r_{1}^{7}}-\frac{70 H z}{r_{2}^{9}}\right) \\
P_{y 2}=\frac{5}{r_{2}^{7}} \cdot y^{2} \\
P_{y 3}=-\frac{1}{r_{1}^{5}} \\
P_{y 4}=-\frac{1}{r_{2}^{5}} \\
P_{y 5}=\frac{4\left(3 r_{2}+H+z\right)}{r_{2}^{3}\left(r_{2}+H+z\right)^{3}}-\frac{4 y^{2}}{r_{2}^{5}\left(r_{2}+H+z\right)^{2}}-\frac{8\left(2 r_{2}+H+z\right)}{r_{2}^{4}\left(r_{2}+H+z\right)^{4}} \cdot y^{2} .
\end{array}\right.
$$


In Equation (20), when $t=0$, the normal strain in $Y$ axis $\varepsilon_{y y}(r, 0)$ is given by

$$
\varepsilon_{y y}(r, 0)=\frac{3 V \tau x y}{8 \pi}\left\{M_{y 0}+M_{y 1}+M_{y 1}^{\prime \prime}+M_{y 2}+M_{y 3}+M_{y 4}\right\} .
$$

The analytic expression of the viscoelastic normal strain $\varepsilon_{z z}(r, t)$ of a point $(x, y, z)$ in $Z$ axis is

$$
\begin{gathered}
\varepsilon_{z z}(r, t)=\frac{3 V \tau x y}{8 \pi}\left\{M_{z 0} H(t)+\left(M_{z 1}+M_{z 1}^{\prime \prime}+t M_{z 1}^{\prime}\right) \exp \left(-\frac{q_{0}}{q_{1}} t\right)+M_{z 2} \exp \left(-\frac{1}{p_{1}^{\prime}} t\right)\right. \\
\left.+M_{z 3} \exp \left(-\frac{3 K+2 q_{0}}{3 K p_{1}+2 q_{1}} t\right)+M_{z 4} \exp \left(-\frac{6 K+q_{0}}{6 K p_{1}+q_{1}} t\right)\right\}
\end{gathered}
$$

where the parameters $M_{z i}$ are as follows:

$$
\left\{\begin{aligned}
M_{z 0}= & 2\left(\frac{q_{0}^{\prime}}{q_{0}}-1\right) \cdot\left[\frac{S_{\varepsilon z z}(0)}{q_{0}\left(3 K+q_{0}\right)}+\frac{3 P_{z 3}}{6 K+q_{0}}\right] \\
M_{z 1}= & \frac{2\left(1-p_{1} T_{1}\right)}{q_{0}} \\
& \times\left\{\frac{S_{\varepsilon z z}\left(T_{1}\right)}{\left(3 K+2 q_{0}\right)-\left(3 K p_{1}+2 q_{1}\right) T_{1}}-\frac{\left(1-p_{1} T_{1}\right)}{1-p_{1}^{\prime} T_{1}} \cdot \frac{3\left(q_{0}^{\prime}-q_{1}^{\prime} T_{1}\right) P_{z 3}}{\left(6 K+q_{0}\right)-\left(6 K p_{1}+q_{1}\right) T_{1}}\right\} \\
M_{z 2}= & -2\left(1-p_{1} T_{2}\right)^{2} \frac{q_{0}^{\prime}-q_{1}^{\prime} T_{2}}{q_{0}-q_{1} T_{2}} \\
& \times\left\{\frac{S_{\varepsilon z z}\left(T_{2}\right)}{\left(q_{0}-q_{1} T_{2}\right)\left[\left(3 K+2 q_{0}\right)-\left(3 K p_{1}+2 q_{1}\right) T_{2}\right]}+\frac{\left(6 K+q_{0}\right)-\left(6 K p_{1}+q_{1}\right) T_{2}}{\left(6 P_{z 3}\right.}\right\} \\
M_{z 3}= & -\frac{2\left(1-p_{1} T_{3}\right)}{\left(3 K+2 q_{0}\right)\left(q_{0}-q_{1} T_{3}\right)}\left[\frac{1-p_{1} T_{3}}{1-p_{1}^{\prime} T_{3}} \cdot \frac{q_{0}^{\prime}-q_{1}^{\prime} T_{3}}{q_{0}-q_{1} T_{3}}-1\right] \cdot S_{\varepsilon z z}\left(T_{3}\right) \\
M_{z 4}= & -\frac{6\left(1-p_{1} T_{4}\right)}{6 K+q_{0}}\left[\frac{1-p_{1} T_{4}}{1-p_{1}^{\prime} T_{4}} \cdot \frac{q_{0}^{\prime}-q_{1}^{\prime} T_{4}}{q_{0}-q_{1} T_{4}}-1\right] \cdot P_{z 3} \\
M_{z 1}^{\prime \prime}= & 2 \cdot\left\{P_{z 1} \cdot S\left(6 K+q_{0}, 6 K p_{1}+q_{1}\right)+P_{z 2} \cdot S\left(6 K+7 q_{0}, 6 K p_{1}+7 q_{1}\right)\right\} \\
M_{z 1}^{\prime}= & -\frac{\left.q_{1}^{\prime} T_{1}\right)\left(1-p_{1} T_{1}\right)^{2}}{q_{0} q_{1}\left(1-p_{1}^{\prime} T_{1}\right)\left[\left(3 K+2 q_{0}\right)-\left(3 K p_{1}+2 q_{1}\right) T_{1}\right]} \cdot S_{\varepsilon z z}\left(T_{1}\right)
\end{aligned}\right.
$$

In Equation (23), $S_{\varepsilon z z}(T)$ is given by

$$
S_{\varepsilon z z}(T)=P_{z 1} \cdot\left[6 K+q_{0}-\left(6 K p_{1}+q_{1}\right) T\right]+P_{z 2} \cdot\left[6 K+7 q_{0}-\left(6 K p_{1}+7 q_{1}\right) T\right] .
$$


For the universal function $S(n, m)$ above see Equations (4) and (5). In Equations (24) and (25), the parameters $P_{z i}$ which related to the coordinate system are as follows:

$$
\left\{\begin{array}{l}
P_{z 1}=-\frac{1}{r_{1}^{5}}+\frac{5(H-z)^{2}}{r_{1}^{7}}+\frac{10 H(2 z+H)}{r_{2}^{7}}-\frac{70 H z(H+z)^{2}}{r_{2}^{9}} \\
P_{z 2}=-\frac{1}{r_{2}^{5}}-\frac{5\left(H^{2}-z^{2}\right)}{r_{2}^{7}} \\
P_{z 3}=-\frac{4}{r_{2}^{5}}
\end{array}\right.
$$

When $t=0$, the normal strain in $Z$ axis $\varepsilon_{\hat{z z}}(r, 0)$ is

$$
\varepsilon_{z z}(r, 0)=\frac{3 V \tau x y}{8 \pi}\left\{M_{z 0}+M_{z 1}+M_{z 1}^{\prime \prime}+M_{z 2}+M_{z 3}+M_{z 4}\right\} .
$$

The viscoelastic analytic expression of the bulk strain $\theta(r, t)$ at a point $(x, y, z)$ is (SoNG et al., 2004)

$$
\begin{aligned}
\theta(r, t)= & \frac{3 V \tau x y}{4 \pi}\left\{M_{\theta 0} H(t)+M_{\theta 1} \cdot \exp \left(-\frac{q_{0}}{q_{1}} t\right)+M_{\theta 2} \exp \left(-\frac{1}{p_{1}^{\prime}} t\right)\right. \\
& \left.+M_{\theta 3} \exp \left(-\frac{3 K+2 q_{0}}{3 K p_{1}+2 q_{1}} t\right)+M_{\theta 4} \exp \left(-\frac{6 K+q_{0}}{6 K p_{1}+q_{1}} t\right)\right\}
\end{aligned}
$$

where the parameters $M_{\theta 0}, M_{\theta 1}, M_{\theta 2}, M_{\theta 3}$ and $M_{\theta 4}$ are as follows

$$
\left\{\begin{array}{l}
M_{\theta 0}=6\left(\frac{q_{0}^{\prime}}{q_{0}}-1\right) \cdot \frac{S_{\theta}(0)}{3 K+2 q_{0}} \\
M_{\theta 1}=-6 \cdot \frac{\left(q_{0}^{\prime}-q_{1}^{\prime} T_{1}\right)}{q_{0}} \cdot \frac{\left(1-p_{1} T_{1}\right)^{2}}{1-p_{1}^{\prime} T_{1}} \cdot \frac{S_{\theta}\left(T_{1}\right)}{\left(3 K+2 q_{0}\right)-\left(3 K p_{1}+2 q_{1}\right) T_{1}} \\
M_{\theta 2}=-6 \cdot \frac{q_{0}^{\prime}-q_{1}^{\prime} T_{2}}{q_{0}-q_{1} T_{2}} \cdot \frac{\left(1-p_{1} T_{2}\right)^{2}}{\left(3 K+2 q_{0}\right)-\left(3 K p_{1}+2 q_{1}\right) T_{2}} \cdot S_{\theta}\left(T_{2}\right) \\
M_{\theta 3}=-6 \cdot \frac{\left(1-p_{1} T_{3}\right)}{\left(3 K+2 q_{0}\right)}\left[\frac{1-p_{1} T_{3}}{1-p_{1}^{\prime} T_{3}} \cdot \frac{q_{0}^{\prime}-q_{1}^{\prime} T_{3}}{q_{0}-q_{1} T_{3}}-1\right] \cdot S_{\theta}\left(T_{3}\right) \\
M_{\theta 4}=-6 \cdot \frac{\left(1-p_{1} T_{4}\right)}{6 K+q_{0}} \cdot \frac{\left(6 K+7 q_{0}\right)-\left(6 K p_{1}+7 q_{1}\right) T_{4}}{\left(3 K+2 q_{0}\right)-\left(3 K p_{1}+2 q_{1}\right) T_{4}} \cdot\left[\frac{1-p_{1} T_{4}}{1-p_{1}^{\prime} T_{4}} \cdot \frac{q_{0}^{\prime}-q_{1}^{\prime} T_{4}}{q_{0}-q_{1} T_{4}}-1\right] \cdot P_{\theta 2}
\end{array}\right.
$$


where the universal function of bulk strain $S_{\theta}(T)$ is given by

$$
S_{\theta}(T)=P_{\theta 1}+P_{\theta 2} \cdot \frac{\left(6 K+7 q_{0}\right)-\left(6 K p_{1}+7 q_{1}\right) T}{\left(6 K+q_{0}\right)-\left(6 K p_{1}+q_{1}\right) T},
$$

where the parameters $P_{\theta 1}$ and $P_{\theta 2}$ which are related to the coordinate system are

$$
\left\{\begin{array}{l}
P_{\theta 1}=\frac{1}{r_{1}^{5}}-\frac{10 H(H+Z)}{r_{2}^{7}} \\
P_{\theta 2}=\frac{1}{r_{2}^{5}}
\end{array} .\right.
$$

When $t=0$, the bulk strain $\theta(r, 0)$ is

$$
\theta(r, 0)=\frac{3 V \tau x y}{4 \pi}\left\{M_{\theta 0}+M_{\theta 1}+M_{\theta 2}+M_{\theta 3}+M_{\theta 4}\right\}
$$

\section{Characteristics of Bulk Strain on the Ground Surface}

In the earthquake precursors, there are many earthquake observation parameters concerning bulk strain, such as geo-electricity, water level below the ground surface, radon content and so on (MEI et al., 1993). We study mainly the bulk strain produced by the spherical inclusion on the ground surface.

\section{The Property of Bulk Strain Function and the Estimation of Medium Parameters}

In the bulk strain expression (32), the difference of parameters $q_{0}, q_{1}, p_{1}, q_{0}^{\prime}, q_{1}^{\prime}, p_{1}^{\prime}$ for the medium rheologic model (SoNG et al., 2003) will produce the difference of the parameters $T_{i}(i=1,2,3,4)$, which determine the time history of bulk strain from initial state to asymptotic value. The parameters $M_{\theta 0}, M_{\theta 1}, M_{\theta 2}, M_{\theta 3}, M_{\theta 4}$ are related to the coordinate and the medium rheologic constants. These parameters define the space field of bulk strain.

When $t<0$, for the boundary condition, $\tau H(t)$ is zero where $H(t)$ is the unit step function and $\tau$ is the shear stress. When $\mathrm{t}>0$, the $\tau H(t)$ is $\tau$ which is given as $10^{8} \mathrm{~Pa}$ (DobrovolsKII,1991) in this paper. In the expression of the bulk strain $\theta(x, y, z, t)$, if $z$ is zero, and $r_{1}=r_{2}=r=\left(x^{2}+y^{2}+H^{2}\right)^{1 / 2}$, where $H$ is the depth of the earthquake focus, $\theta(x, y, 0, t)$ is the expression of the bulk strain concerning the $t$ on the random point of the ground surface. We may calculate the perturbation of bulk strain $\Delta \theta$ as $\Delta \theta(x, y, 0, t)=\theta(x, y, 0, t)-\theta(x, y, 0,0)$. Then we may analyze the characteristics of the time history and the space distribution of bulk strain. 
As to how to choose the elastic and rheologic constants of the medium (DobrovolskiI, 1991) given $\mu=2 \times 10^{4} \mathrm{MPa}$ for the shear modulus of the Earth's crust, the Poisson's ratio $v=0.25$. ZHANG (1987) given $E=10^{5} \mathrm{MPa}$ for the Young's modulus of the Earth's crust, viscosity constant $\eta=10^{22} \mathrm{~Pa}$ s. WANG (1979) and MEI et al. (1992) took viscosity constant $10^{17} \sim 10^{23} \mathrm{~Pa} \mathrm{~s}$ and the Young's modulus of the Earth's crust $10^{4} \sim 10^{5} \mathrm{MPa}$. KASAHARA (1979) took the viscosity constant $\eta=2 \times 10^{21} \mathrm{~Pa}$ s. MATSU'URA et al. (1999) and FuKAHATA et al. (2004) given the viscosity of $5 \times 10^{18} \mathrm{~Pa}$.

Having referenced the above results, in this paper we take the Poisson's ratio of the rock medium of 0.25 for both in and outside the inclusion, and the viscosity constants and the Young's modulus of the medium outside the inclusion of $10^{21} \mathrm{~Pa} \mathrm{~s}$ and $10^{5} \mathrm{Mpa}$, respectively. The parameters of the hard inclusion and the soft one are shown in the Table 1.

Based on the relation of parameters of the rheologic medium model (SoNG et al., 2003) as follows, we obtain the parameters $q_{0}, q_{1}, p_{1}, q_{0}^{\prime}, q_{1}^{\prime}, p_{1}^{\prime}$ and the bulk strain by the expression (32).

$$
\left\{\begin{array} { l } 
{ p _ { 1 } = \frac { \eta } { E _ { 1 } + E _ { 2 } } } \\
{ q _ { 0 } = \frac { E _ { 1 } \cdot E _ { 2 } } { E _ { 1 } + E _ { 2 } } } \\
{ q _ { 1 } = \frac { E _ { 1 } \eta } { E _ { 1 } + E _ { 2 } } }
\end{array} \quad \left\{\begin{array}{l}
p_{1}^{\prime}=\frac{\eta^{\prime}}{E_{1}^{\prime}+E_{2}^{\prime}} \\
q_{0}^{\prime}=\frac{E_{1}^{\prime} \cdot E_{2}^{\prime}}{E_{1}^{\prime}+E_{2}^{\prime}} \\
q_{1}^{\prime}=\frac{E_{1}^{\prime} \eta^{\prime}}{E_{1}^{\prime}+E_{2}^{\prime}}
\end{array}\right.\right.
$$

where the situation of considerably smaller $\eta$ corresponds mainly to the elastic medium, whereas the very great $\eta$ corresponds mainly to the viscoelastic and the case of moderate $\eta$ represents the elastic-viscoelastic rheologic medium. Therefore, the parameter selection for the rheologic medium model is very important.

The $\eta$ value selection may influence the time constants $T_{i}(i=1,2,3,4)$ of viscosity. For the linear model, with a different physical mechanism or a different $\eta$ or $T_{i}$, the scale of time may be varied, but its conclusion may still be accepted in principle.

Table 1

Parameters of rheologic medium model in and outside the inclusion

\begin{tabular}{lccc}
\hline Parameter & Wall rock & Hard inclusion & Soft inclusion \\
\hline$E_{1}$ or $E_{1}^{\prime}(\mathrm{MPa})$ & $E_{1}=1 \times 10^{5}$ & $E^{\prime}{ }_{1}=1.5 \times 10^{5}$ & $E^{\prime}{ }_{1}=0.5 \times 10^{5}$ \\
$E_{2}$ or $E_{2}(\mathrm{MPa})$ & $E_{2}=1 \times 10^{5}$ & $E_{2}^{\prime}=1.5 \times 10^{5}$ & $E_{2}^{\prime}=0.5 \times 10^{5}$ \\
$\eta$ or $\eta^{\prime}(\mathrm{Pa} \mathrm{s})$ & $\eta=1 \times 10^{21}$ & $\eta^{\prime}=2 \times 10^{21}$ & $\eta^{\prime}=0.5 \times 10^{21}$ \\
\hline
\end{tabular}


Assuming both the radius and depth of inclusion of $25 \mathrm{~km}$, we may analyze the space-time evolution characters for bulk strain on the ground $(z=0)$ produced by the hard inclusion or the soft one.

\section{Quadrant of Bulk Strain Produced by a Hard Inclusion and a Soft One}

The characteristics of the bulk strain spatial field of the hard inclusion DoBrovolsKII (1991) and the soft one BRADY (1974) are similar in that both have quadrant distribution and the distribution inside the region with the size two times the extension of seismic source is contrary to the one outside this region. The dissimilarity is that the bulk strain high-value regions produced by the hard inclusion correspond rightly to the low-value regions of the soft inclusion.

Pattern features of the bulk strain versus time. Pattern features of the bulk strain versus time produced by a hard inclusion are as follows: (1) The bulk strain pattern varies with the distance to the epicenter. The bulk strain in the source region goes through a process of "increasing $\rightarrow$ maximum $\rightarrow$ decreasing $\rightarrow$ tend towards a stable value" and seems like an upward protruding (Fig. 2). For the points around the region with its distance to seismic foci about two times the extension of seismic source, their bulk strains go down initially, then ascend and show the characteristics of "N".

The bulk strains at the points outside the region with the size two times the extension of seismic source show the characteristics of downward protrution. The

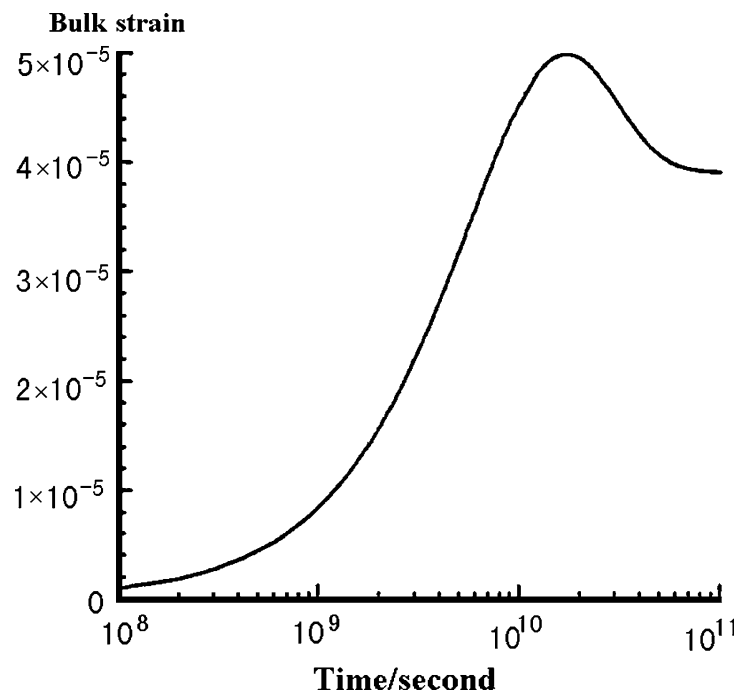

Figure 2

Bulk strain curve versus time in the near-source region of the hard inclusion. 
patterns at points distant from the epicenter are basically similar to the seismic source, yet their variation values are comparatively smaller. (2) The variation value of bulk strain decreases as the distance increases.

It is meaningful that the pattern of bulk strain produced in the soft inclusion is different from that in the hard inclusion. The bulk strain values produced in the soft inclusion all increase gradually with the different epicenter distances, then tend towards a stable value, however there is no decreasing process after the maximum value.

\section{Three-stage Characteristics of the Bulk Strain Produced in a Hard Inclusion}

The bulk strain in the hard inclusion possesses three-stage characteristics of "increasing $\rightarrow$ maximum $\rightarrow$ decreasing to a stable value." That is, during the stage $\alpha$, the bulk strain value increases gradually with the passage of time and the bulk strains in the near-source region and the far-source region all show outward extension. During the stage $\beta$, the bulk strain value and its range all vary slightly with time and reach an extreme state. Other relevant physical parameters such as strain, stress, etc. should be extreme too, then earthquake precursors during the stage $\beta$ should be most significant and occur at a similar time in the near-source and far-source regions. During the stage $\gamma$, the bulk strain value weakens as time continues, and the corresponding spatial characteristics are that the bulk strain of the far-source region shows contraction to the epicenter and the bulk strain of the near-source area migrates outwards.

\section{Characteristics of Bulk Strain Produced in a Soft Inclusion}

The study shows that the bulk strains in the near-source region and the far-source area produced in the soft inclusion all diffuse outwards and their diffusing directions are conjugate. If a soft inclusion is regarded as an earthquake preparation model, the spatial-temporal evolution of precursors should diffuse outwards in the whole seismic preparation process.

\section{Application of Theoretical Result to Earthquake Precursors}

There exist three stages (namely $\alpha, \beta$ and $\gamma$ ) in the process of bulk strain versus time in the near-source region caused by the hard inclusion (Fig. 2). Having studied abundant observation data of crust deformation, Mesherikov (1968) and FuJII (1974) found that the strain curves have characteristics of three stages in the earthquake preparation process, namely, $\alpha, \beta$ and $\gamma$ (Fig. 3). Though our theoretical curves cannot give the relative proportion of the three stages, the similarity of basic patterns of the theoretical and empirical curves supports the scientific reliability of 


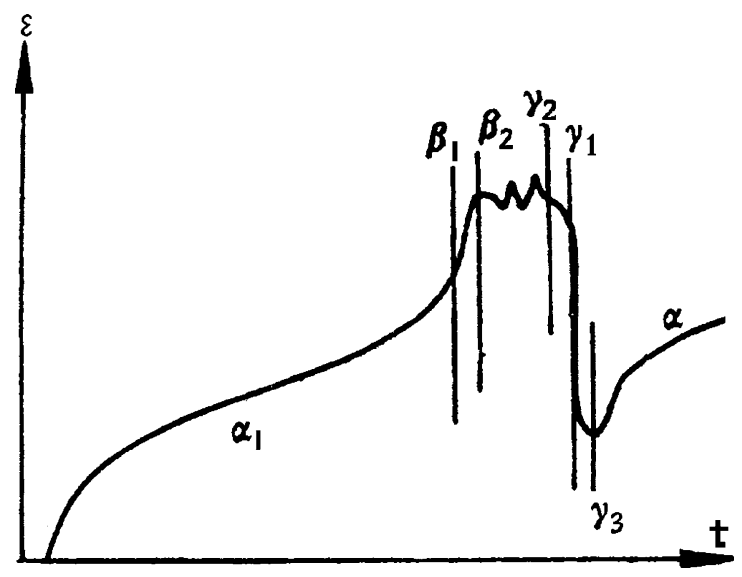

Figure 3

The three stages $(\alpha, \beta$ and $\gamma$ ) of strain (Mesherikov, 1968; FuJII, 1974).

the theoretical curves. This also supports the rationality of the strong-body earthquake preparation model.

The bulk strain curve pattern of the hard inclusion in the near-source region is "small $\rightarrow$ big $\rightarrow$ maximum $\rightarrow$ decreasing to stability" and shows an upward protruding variation. At points around the region with its distance to foci about two times the extension of seismic source, the pattern of bulk strain curves is "descending $\rightarrow$ ascending" and shows the characteristic of "N" type. The bulk strain types at points near the region of three times the extension of seismic source show downward protruding variation. The bulk strain at points distant from the inclusion has little variation. These are similar to the phenomena in the earthquake observation in that the bulk strain abnormal types vary with the increase of the distance (MEI et al., 1993). In other words, this phenomenon does not occur occasionally, but possesses a physical basis.

LI (1981) found that the water-radon anomalies exhibit the development pattern outward half a year before the Tangshan earthquake. ZHENG (1979) had studied filtered water-level data and found that the water-level descending anomalies develop outwards in the directions of north-east and north-west a half year before the Tangshan earthquake. There also exist the characteristics about a half year before the Longling earthquake and the Songpan shock (MEI et al., 1993). Song's studies (SoNG et al., 2000) reveal that the three stages $(\alpha, \beta$ and $\gamma)$ are very evident. Particularly, there all exist the subphase $\gamma_{1}$ of precursors contracting from the periphery to the epicenter region and the subphase $\gamma_{2}$ of precursors diffusing from the epicenter area to the periphery during the $\gamma$-stage (corresponding to earthquake short-term and imminent-term stage). It can be seen that the existence of $\alpha-\beta$ - and $\gamma$-stage and the spatial-temporal migration of precursors may be controlled by the earthquake 
preparation body. It may be a normal phenomenon and should be an important basis to predict earthquakes.

Quadrant distribution is one of the common characteristics of the spatial distribution of bulk strain on the ground with differences in some details associated with earthquake preparation. The spatial range and the values of the anomaly in bulk strain all increase with time and decrease after reaching the maximum value in the model of hard inclusion. For the soft model there is only trend variation, however. Experimental results (XU et al., 1997) show compatible quadrant distribution. In earthquake precursors there exist some consistent quadrant distribution of anomalies in the deformation and georesistivity before the cases of Datong earthquake, Zhangbei earthquake and Baotou earthquake, etc. (SoNG et al., 2001). This feature would be useful for predicting the location of a strong earthquake.

By earthquake precursors, we refer to the phenomena which may occur before larger earthquakes. MeI et al. (1993 and 1994) elaborated the physical processes of earthquake generation. This paper will not describe it in details.

\section{Conclusions}

To date we have described the derivation of the bulk strain and ground deformation and the preliminary application based on the theory of a rheologic inclusion model in the seismogenic process. The results are as follows:

Based on the Correspondence Principle, we obtained the analytic expression of the viscoelastic displacement $U(r, t), V(r, t)$ and $W(r, t)$, the viscoelastic normal strains $\varepsilon_{x x}(r, t), \varepsilon_{y y}(r, t)$ and $\varepsilon_{z z}(r, t)$ and the bulk strain $\theta(r, t)$ produced a threedimension inclusion in the semi-infinite rheologic medium defined by a standard linear rheologic model.

Applying the theoretical expression of the three-dimension rheologic inclusion model, we throughly computed the spatial-temporal evolution process of bulk strain on the ground produced by a spherical rheologic inclusion DoBRovolSKII (1991) in the semi-infinite rheologic medium. The results show that spatialtemporal evolution of bulk strain produced by a hard inclusion has three stages of different characteristics, which are similar to most of the geodetic deformation curves, but not for the case of soft inclusion. The $\alpha$-stage characteristic is a long stage in which the precursors in both the near-field and the far-field develop from the focal region to the periphery. The $\beta$-stage shows a very rapid propagation of the precursors, in which they appear everywhere. During the $\gamma$-stage, the precursors in the far-field converge from the periphery, and the precursors in the near-field develop outwards.

The theoretical results have been applied to explain tentatively the stage variation of the spatial-temporal evolution, the pattern feature and quadrant-distribution 
characteristics of earthquake precursors. It is conducive to better understand the process of seismogeny and occurrence, and to construct the physical model of the development of earthquakes.

Questions remain to be studied, for example, the viscoelastic expression of geoelectricity, geomagnetism, wave-velocity and so on.

\section{Acknowledgements}

Gratefull acknowledgment the support from the Chinese Joint Seismological Foundation (101105) and Committee of Science and Technology of Shanghai Municipality, NSFC under grant: 10232050, MOST (Ministry of Science and Technology, China, Grant No. 2004 CB418406) and Computer Network Information Center, CAS under grant INF105-SCE-2-02.

\section{REFERENCES}

Brady, B. T. (1974), Theory of earthquake, Pure Appl. Geophys. 112(4), 701-725.

Dobrovolskii, I. P., Theory on the Preparation of Tectonic Earthquakes (in Russian), Ivz. An SSSR (Moscow, 1991).

EShelbym, J. D. (1957), The determination of the elastic field of an ellipsoidal inclusion and related problem, Proceed. Roy. Soc. A241, 376-396.

FuJI, Y. (1974), Relationship between duration time of anomalous vertical deformation of the crust and magnitude before the occurrence of an earthquake (in Japanese with English abstract), Earthquake 27(3), 197-213.

Fukahata, Y., Nishitani, A., and Matsu'ura, M. (2004), Geodetic data inversion using ABIC to estimate slip history during one earthquake cycle with viscoelastic slip-response functions, Geophys. J. Int. 156, $140-153$.

LI, X. (1981), Phenomena of diffusion and contraction of water-radon precursors (in Chinese with English abstract), Earthquake 1(5), 340-357.

Kasahara, K. (1979), Migration of crust deformation, Tectonophysics 52, 329-341.

Matsu'ura, M., Nishitani, A., and Fukahata, Y. (1999), Slip History during one earthquake cycle at the Nankai Subduction zone, inferred from the inversion analysis of leveling data with a viscoelastic slip response function, $1^{\text {st }}$ ACES Workshop Proceedings (http://quakes.earth.uq.edu.au/ACES/).

MeI, S. R. and ZHU, Y. Q., Earthquake-generating Model of the Tangshan Earthquake (in Chinese) (Seismological Press, Beijing, 1992).

Mei, S., Feng, D., Zhang, G., et al., Introduction to Earthquake Prediction in China (in Chinese) (Beijing: Seismological Press, 1993).

MEI, S. (1994), A study on the physical model of earthquake precursor field and the mechanism of precursors' time-space distribution_Origin and evidences of the strong body earthquake-generating model, Acta Seismologica. Sinica. 7(3), 273-282.

Mesherikov, J. A. (1968), Recent crust movements in seismic region: Geodetic and geomorphic data, Tectonophysics 6, 29-39.

Song, Z. On the Spatial Temporal Evolution of the Load/Unload Response Ratio (LURR) and Other Earthquake Precursors and their Implications (in Chinese with English abstract) (Ph. D. Thesis), Institute of Geophysics, China Seismological Bureau, (Beijing, 1996).

Song, Z., YIN, X., and MEI, S. (2000), Theoretical analysis of the spatio-temporal evolution of the bulk strain field based on a rheologic inclusion model, Acta Seismologica. Sinica. 13(5), 525-535. 
Song, Z., Xu, P., ZHAng, H., et al. (2001), Quadrant characteristics of earthquake precursors and its theoretical analysis, Acta Seismologica. Sinica. 14(1), 58-65.

Song, Z., YIN, X., and MeI, S. (2003), Three-dimensional rheologic model of earthquake preparation (1) displacement field, Acta Seismologica. Sinica. 16(6), 588-597.

Song, Z., YIN, X., and MeI, S. (2004), Three-dimensional rheologic model of earthquake preparation (2) - strain field and its applications, Acta Seismologica. Sinica. 17(2), 131-143.

WANG, R., Base of solid mechanics (in Chinese) (Geological Press, Beijing, 1979).

Xu, Z., WAng, B., Zhao, J., et al. (1997), An experimental study of fractural features of concrete samples containing hard inclusion, Acta Seismologica. Sinica. 10(1), 97-105.

YIN, X. and ZHEN, T. (1982), Rheologic model of earthquake preparation process, Scientia Sinica (series B) 10, 922-930.

YIN, X., Solid Mechanics (In Chinese) (Seismological Press, Beijing, 1985).

ZHANG, G. (1987), Application of rock rheologic model to earthquake-generating process and precursors (in Chinese with English abstract), Acta Seismological. Sinica. 9(4), 384-391.

ZHENG, Z. (1979), On generation process of the Tangshan earthquake basing on water-level filtered data (in Chinese with English abstract), Acta Geophysica. Sinica. 22(3), 267-280.

(Received December 29, 2004, revised September 19, 2005, accepted December 26, 2005)

Published Online First: August 31, 2006

\section{(7) To access this journal online: \\ (20) http://www.birkhauser.ch}

